Communications in Physics, Vol. 21, No. 1 (2011), pp. 1-9

\title{
SEVERAL PHYSICAL PROPERTIES OF TWO INTERACTING COMPLEX SCALAR FIELDS AT FINITE DENSITY
}

\author{
TRAN HUU PHAT \\ Vietnam Atomic Energy Commission \\ PHAN THI DUYEN \\ Department of Physics, Hanoi National University of Education
}

\begin{abstract}
The two interacting complex scalar fields at finite density is considered in the mean field approximation. It is shown that although the symmetry is spontaneously broken for the chemical potentials bigger than the meson masses in vacuum, but the Goldstone theorem is not preserved in broken phase. Then two mesons are condensed and their condensates turn out to be two-gap superconductor which is signaled by the appearance of the Meissner effect as well as the Abrikosov and non-Abrikosov vortices. Finally, there exhibits domain wall which is the plane, where two condensates flowing in opposite directions collide and generate two types of vortices with cores in the wall. and domain wall.
\end{abstract}

\section{INTRODUCTION}

As was known, the evolution of modern physics always links closely with scalar fields. They appear in a lot of physical theories. Scalar fields play the role of order parameters in the Landau theory of phase transition [1], in the Ginzburg-Landau theory of superconductivity [2]. Fundamental scalar fields are necessarily present in all unification theories: from the Standard Model of particles to the Grand Unification Model [3, 4], here scalar fields describe the Higgs bosons. In superstring theory scalar field emerges in the form of dilaton and in Cosmology scalar fields are needed in the inflation theory $[5,6]$. However, all the above mentioned theories of scalar fields have been considered in vacuum only. It is expected that in medium these fields manifest many other interesting features which are absent in vacuum. In effect, it is well known that the condensation of kaons and charged pions in dense matter and compact stars has been the interesting subject since long ago [7-10]. Bose-condensed states are expected to be found in the interior of compact stars with density around $3 \rho_{0}$, where $\rho_{0}$ is normal nuclear density. Until now we are aware of only few points in the $T-\rho$ plane, namely, the vacuum $(T=0, \rho=0)$ and nuclear $\operatorname{matter}\left(T=0, \rho=\rho_{0}=0.17 \mathrm{fm}^{-3}\right)$. Therefore, there are much experimental and theoretical efforts to get information about other regions. In recent years experimental studies are carried out through observing the matter produced in heavy-ion collisions at intermediate energies, in particular, the nuclear reactions induced by radioactive beams which offer a new opportunity to consider the isospin degree of freedom of asymmetric nuclear matter. As a consequence, the theoretical researches of Quantum Chromodynamics (QCD) at finite baryon density and isospin chemical potential are intensively implemented. It was shown 
that in the color-flavor-locked phase kaon condensation occurs at high baryon density and low temperature [11] and the lattice simulation of QCD at finite isospin chemical potential [12- 14] proved that there occur the Bose-Einstein condensation of charged pions at high isospin density and low temperature. In this connection, investigating simplified models of strongly interacting matter, such as the Nambu-Jona-Lasinio model [15 - 18], the linear sigma model [19-21], the chiral perturbation theory [22-25] etc, is very important for the understanding of matter states under different conditions. Following this trend we shall consider in this paper the system consisting of two interacting complex scalar fields at finite density. It is well established that the interaction between two species of a multi component system might lead to many new phenomena.

The Lagrangian of the system reads

$$
\begin{aligned}
L & =\sum_{i=2}^{2}\left(\partial_{\mu} \varphi_{i}^{*}\right)\left(\partial^{\mu} \varphi_{i}\right) \varphi_{i}-V_{0} \\
V_{0} & =\sum\left[m_{i}^{2} \varphi_{i}^{*} \varphi_{i}+\frac{\lambda_{i}}{2}\left(\varphi_{i}^{*} \varphi_{i}\right)^{2}\right]+\eta\left[\left(\varphi_{1}^{*} \varphi_{2}\right)^{2}+\left(\varphi_{2}^{*} \varphi_{1}\right)^{2}\right]
\end{aligned}
$$

where $m_{i}$ are the masses of particles, $\lambda$ and $\eta$ are coupling constants, $\lambda_{i}>0$ At finite density (1) becomes

$$
L=\sum\left(\partial_{\mu}+i \mu_{i} \delta_{0 \mu}\right) \varphi_{i}^{*}\left(\partial_{\mu}-i \mu_{i} \delta_{0 \mu}\right) \varphi_{i}-V_{0}
$$

\section{PHYSICAL PROPERTIES}

Starting from the Lagrangian (2) we consider respectively the following physical properties:

1- Spontaneous Symmetry Breaking and Goldstone Theorem

It is evident that (2) is invariant with respect to the phase transformations of the group $U(1)$,

$$
\varphi_{i}=e^{i \alpha} \varphi_{i}, \quad i=1,2 .
$$

The corresponding conserved quantity is the total charges of the system $\rho=\rho_{1}+\rho_{2}$. For $\eta=0$ the symmetry group of $(1 \mathrm{~b})$ is $U(1) \times U(1)$,

$$
\varphi_{j}=e^{i \alpha_{j}} \varphi_{j}, j=1,2 .
$$

The corresponding conserved quantities are separate charges of every complex field. From now on we are interested only to the case $\eta \neq 0$. Then due to the Goldstone theorem there exists only one gapless mode in the broken phase.

Assume that the field operators $\varphi_{1}, \varphi_{2}$ develop the vacuum expectation values $u$ and $v$ which in the tree-level are determined by the minimum conditions

$$
\begin{aligned}
& \frac{\partial V}{\partial u}=-2\left(\mu_{1}^{2}-m_{1}^{2}\right) u+2 \lambda_{1} u^{3}+4 \eta v^{2} u=0 \\
& \frac{\partial V}{\partial v}=-2\left(\mu_{2}^{2}-m_{2}^{2}\right) v+2 \lambda_{2} v^{3}+4 \eta u^{2} v=0
\end{aligned}
$$


Beside the vanishing solutions $\mathrm{u}=\mathrm{v}=0$ the system (3) possesses the non-trivial solutions

$$
\begin{aligned}
& u^{2}=\frac{\lambda_{2}\left(\mu_{1}^{2}-m_{1}^{2}\right)-2 \eta\left(\mu_{2}^{2}-m_{2}^{2}\right)}{\lambda_{1} \lambda_{2}-4 \eta^{2}} \\
& v^{2}=\frac{\lambda_{1}\left(\mu_{2}^{1}-m_{2}^{2}\right)-2 \eta\left(\mu_{1}^{2}-m_{1}^{2}\right)}{\lambda_{1} \lambda_{2}-4 \eta^{2}}
\end{aligned}
$$

The boundedness of the potential requires $\lambda_{1} \lambda_{2}-4 \eta^{2}>0$. Therefore the symmetry breaking of the system are determined by the members in the right hand side of Eqs. (4), which are respectively denoted by $f\left(\mu_{1}, \mu_{2}\right), g\left(\mu_{1}, \mu_{2}\right)$.

If $f, g>0$ the symmetry group $U(1)$ of both fields is spontaneously broken and, consequently, both bosons are condensed. In the case when one of these two function is negative, say, g, then the boson corresponding to $\varphi_{1}$ is the only one which is condensed. For convenience, in what follows it is supposed that both functions are positive. The vacuum manifold $\mathrm{M}$ of the theory consists of many submanifolds:

$$
\begin{aligned}
& M_{1}=\left(u_{+}=+\sqrt{f}, v_{+}=+\sqrt{g}\right) \\
& M_{2}=\left(u_{-}=-\sqrt{f}, v_{-}=-\sqrt{g}\right) \\
& M_{3}=\left(u_{+}, v_{-}\right) \\
& M_{4}=\left(u_{-}, v_{+}\right)
\end{aligned}
$$

Conventionally we choose the vacuum state of the theory corresponds to $M_{1}$ then the symmetry is spontaneously broken, moreover, this manifold can be treated as product of two circles

$$
M_{1}=S^{1} x S^{1}
$$

if we identify $u_{+}=\left|\varphi_{1}\right|, v_{+}=\left|\varphi_{2}\right|$. However, as we will indicate in the next subsection, in the ground state the phases of these fields are not independent, and moreover, due to the constraint between two phases there are not only one pair $\left(u_{+}, v_{+}\right)$in the ground state, but two pairs $\left(u_{1+}, v_{1+}\right)$ and $\left(u_{2+}, v_{2+}\right)$ therefore, in reality the vacuum manifold consists of two disconnected circles, namely,

$$
\begin{aligned}
& M_{1}=S^{1} \\
& M_{2}=S^{1}
\end{aligned}
$$

Next the field operators are shifted

$$
\varphi_{j}=u+\psi_{j}+i \chi_{j}, j=1,2 .
$$

Inserting (7) into (2) leads the new expression for the Lagrangian and there from we find the inverse propagators

$$
\begin{array}{r}
i D^{-1}(k)=\left\|A_{i k}\right\| \\
A_{11}=\omega^{2}-\vec{k}^{2}+2 \lambda_{1} u^{2}, \\
A_{22}=\omega^{2}-\vec{k}^{2}-4 \eta v^{2} \\
A_{12}=A_{21}=2 i \mu_{1} \omega,
\end{array}
$$


and

$$
\begin{array}{r}
i G^{-1}(k)=\left\|B_{i k}\right\|, \\
B_{11}=\omega^{2}-\vec{k}^{2}+2 \lambda_{2} v^{2}, \\
B_{22}=\omega^{2}-\vec{k}^{2}-4 \eta u^{2}, \\
B_{12}=B_{21}=2 i \mu_{2} \omega .
\end{array}
$$

The spectra of meson energies are given by

$$
\begin{aligned}
& \operatorname{det} D^{-1}(k)=0 \\
& \operatorname{det} G^{-1}(k)=0
\end{aligned}
$$

which do not yield any gapless mode in the broken phase.

This implies that the model (1) does not preserve the Goldstone theorem at tree level.

\section{2- Meissner Effect and Two-gap Superconductors}

To proceed further let us consider the system in the presence of an electromagnetic field $A_{\mu}$. The Lagrangian 1 (1) is then replaced by

$$
L=\sum\left(\partial_{\mu}+i \mu_{j} \delta_{0 \mu}+i e A_{\mu}\right) \varphi_{j}^{*}\left(\partial^{\mu}-i \mu_{j} \delta_{0 \mu}-i e A^{\mu}\right) \varphi_{j}-V_{0}-\frac{1}{4} F_{\mu \nu} F^{\mu \nu}
$$

Substituting the decompositions

$$
\varphi_{1}=u+\Phi_{1} ; \varphi_{2}=v+\Phi_{2}
$$

into (10) and then varying the resulting Lagrangian respect to $A_{\mu}$ we are led to the equations for the electromagnetic field

$$
\left(\square-e^{2} u^{2}-e^{2} v^{2}\right) A_{\mu}=j_{\mu}
$$

here $j_{\mu}$ is the supercurrent,

$$
\begin{aligned}
j_{\mu}= & j_{1 \mu}+j_{2 \mu}, \\
j_{a \mu}= & i e\left(\Phi_{a} \partial_{\mu} \Phi_{a}^{*}-\Phi_{a}^{*} \partial_{\mu} \Phi_{a}\right)+i e\left(\partial_{\mu} \Phi_{a}^{*}-\partial_{\mu} \Phi_{a}\right) \\
& -e^{2} u\left(\Phi_{a}^{*}+\Phi_{a}\right)-e^{2} \Phi_{a}^{*} \Phi_{a}
\end{aligned}
$$

$a=1,2$.

Eqs. (11) shows that the photon acquires a mass

$$
m_{p h}=e \sqrt{u^{2}+v^{2}}
$$

in broken phase. This is exactly the Meissner effect in superconductors which tells that the magnetic field can only penetrate into the superconductor to a depth L,

$L \approx \frac{1}{m}_{p h}$ in the unit system where $\hbar=c=1$. It is very interesting to note that in Eqs. (11), (12), and (13) there involve the contributions of two meson condensates. This is the so-called two-gap superconductor which is the hot topic of modern condensed physics [26]. The two-gap superconductors turn out to be conventional superconductors if one of the bosons is not condensed.

\section{3-Abrikosov and non-Abrikosov Vortices}


Next let us proceed to the vortices occurring in the presence of an electromagnetic field. To this end, in the cylindrical system of coordinates $(\rho, \theta, z)$ we look for the radially symmetric solutions with finite energy per unit length, which defines the infinite manifold

$$
M_{\infty}=\rho=R, R \rightarrow \infty \text {. }
$$

From (1) it is obvious that the energy density per length $\mathrm{L}$ is determined by

$$
E / L=\int d x d y\left[\sum_{\substack{k=1,2 \\ j=1}}^{2}\left\{\left(D_{k} \varphi_{j}\right)^{*}\left(D_{k} \varphi_{j}\right)+U\right\}+\frac{\vec{B}^{2}}{8 \pi^{2}}\right],
$$

here $D_{k}=\partial_{k}-i e A_{k}, U=V\left(\varphi_{1}, \varphi_{2}\right)-V(u, v), \vec{B}=\operatorname{curl} \vec{A}$

In order for (14) to be finite it is required that when $\rho=R \rightarrow \infty$

$$
\begin{aligned}
\left|\varphi_{1}\right| \rightarrow u,\left|\varphi_{2}\right| & \rightarrow v, \\
\left|D_{k} \varphi_{j}\right| & \rightarrow 0
\end{aligned}
$$

It is clear that (15) establish the map from $M_{\infty}\{\rho=R \rightarrow \infty\}$ to the ground state $M_{i}, i=1,2$, that is the map: $S^{1} \rightarrow S^{1}$ that possesses the non-trivial fundamental group $\pi_{1}\left(S^{1}\right)=Z$. Consequently we have an infinite number of vortices adopting the following ansatz

a-The first type of vortices

$$
\begin{aligned}
\varphi_{1} & =u_{1} f_{1}(\rho) e^{i m \theta(x)} \\
\varphi_{2} & =v_{1} g_{1}(\rho) e^{i m \theta(x)} \\
A_{\mu} & =\frac{m}{e} A^{1}(\rho) \partial_{\mu} \theta(x)
\end{aligned}
$$

b-The second type of vortices

$$
\begin{aligned}
\varphi_{1} & =u_{2} f_{2}(\rho) e^{i n \theta(x)} \\
\varphi_{2} & =v_{2} g_{2}(\rho) e^{i n \theta(x)} \\
A_{\mu} & =\frac{n}{e} A^{2}(\rho) \partial_{\mu} \theta(x)
\end{aligned}
$$

The boundary conditions read

$$
\begin{aligned}
& f_{i}(0)=g_{i}(0)=A^{i}(0)=0 \\
& \text { and } \\
& f_{i}(\infty)=g_{i}(\infty)=A^{i}(\infty)=1
\end{aligned}
$$

As will be shown later these two types of vortices are trapped in the domain walls and then form a vortex sheet. We especially focus on the following interesting of the vortices:

- When $\Delta \alpha=\Delta \beta=2 \pi n, n=0,1,2,3, \ldots$ vortices have finite energy [27] 
-When $\Delta \alpha=2 \pi m, \Delta \beta=2 \pi n$ vortices not only possess finite energy but also carry fractional magnetic flux $[26,27]$,

$$
\begin{aligned}
& \Phi_{(m, n)}=\left[m \sin ^{2} \theta+n \cos ^{2} \theta\right] \Phi_{0} \\
& \Phi_{0}=\frac{\hbar c}{e}
\end{aligned}
$$

Then we have non-Abrikosov vortices. This is an important feature of two-gap superconductors.

\section{4-Domain Walls}

Let us first remark that on the length scale much larger than the correlation lengths the only important degrees of freedom are the phases of two fields $\varphi_{1}, \varphi_{2}$, whose amplitudes can be regarded as frozen. The energy of the system (1) reads

$$
E=\int d x\left[u^{2}\left(\partial_{\mu} \alpha\right)^{2}+v^{2}\left(\partial_{\mu} \beta\right)^{2}-2 \eta u v \cos (\alpha-\beta)\right]
$$

The energy potential $2 \eta u v \cos (\alpha-\beta)$ has its minimum at $\theta=\alpha-\beta=0$ for $\eta<0$ and $\theta=\alpha-\beta=\frac{\pi}{2}$ for $\eta>0$. The domain wall we are searching for corresponds to the local minimum of the energy potential of (1),

$$
\begin{aligned}
& -\left(\mu_{1}^{2}-m_{1}^{2}\right) u+\lambda_{1} u^{3}+2 \eta v^{2} u e^{2 i(\beta-\alpha)}=0 \\
& -\left(\mu_{2}^{2}-m_{2}^{2}\right) v+\lambda_{2} v^{3}+2 \eta u^{2} v e^{2 i(\alpha-\beta)}=0
\end{aligned}
$$

where $\varphi_{10}=u e^{i \alpha}, \varphi_{20}=v e^{i \beta},\left(\varphi_{10}, \varphi_{20}\right) \in M$, M is the vacuum manifold.

Eqs. (17) possess real solutions only if

$$
\theta=\alpha-\beta=0(\bmod 2 \pi) \text { and } \theta=\alpha-\beta=\frac{\pi}{2}(\bmod 2 \pi)
$$

which correspond to global minimum of the energy $\mathrm{E}$ (16) for $\eta<0$ and $\eta>0$, respectively. Accordingly, Eqs. (17) give two pairs $\left(u_{i}, v_{i}\right), i=1,2$. Thus the ground state of our model is degenerate with respect to the relative phase of two fields. Since $\theta$ is defined only modulo $2 \pi$ all different domains need not be separated by the walls. tions

Varying (16) with respect to $\varphi_{1}$ and $\varphi_{2}$, respectively, we arrive at the following equa-

$$
u^{2} \alpha=-v^{2} \beta=-2 \eta u v \sin 2(\alpha-\beta)
$$

It is obvious that Eqs. (18) reduce to the well-known sine-Gordon equations for the domain wall solution of only one coordinate, say, $z$,

$$
\frac{d^{2} \theta}{d z^{2}}+2|\eta| \frac{u^{2}+v^{2}}{u v} \sin 2 \theta=0
$$

It is known that the non-trivial solution to Eq. (19) is

$$
\begin{aligned}
\theta & =\arctan e^{k z}, \\
k^{2} & =2|\eta| \frac{u^{2}+v^{2}}{u v}
\end{aligned}
$$

changing from 0 to $2 \pi$ as $\mathrm{z}$ runs from $-\infty$ to $+\infty$. The thickness of the wall is $\delta=k^{-1}$ and its energy is finite. 
From the relation

$$
\alpha=\frac{u^{2}}{u^{2}+v^{2}} \theta, \beta=-\frac{v^{2}}{u^{2}+v^{2}} \theta
$$

it follows that the ground state in both sides of the domain wall are different: at $\mathrm{z}=$ $-\infty, \alpha=\beta=0$, whereas at $z=+\infty$ we obtain $\alpha=\pi \frac{v^{2}}{u^{2}+v^{2}}, \beta=-\pi \frac{u^{2}}{u^{2}+v^{2}}$, that is $\theta=\alpha-\beta=\pi$. This implies that as one passes through the wall $\theta$ changes by $\pi$.

Domain walls can contain vortices on which the phases of fields are undefined and, therefore, either $\varphi_{1}(t, x, y, z)$ and/or $\varphi_{2}(t, x, y, z)$ vanish, namely, for fixed $t$,

$$
\begin{aligned}
& \psi_{1}(t, x, y, z)=0 \\
& \chi_{1}(t, x, y, z)=0
\end{aligned}
$$

and / or

$$
\begin{gathered}
\psi_{2}(t, x, y, z)=0 \\
\chi_{2}(t, x, y, z)=0
\end{gathered}
$$

at any point $P(x, y, z)$ of the vortex.

It is indicated [28] that Eqs. (22), (23) determine two types of vortices: the first vortices associated with (18a) and the second vortices with (18b). In effect, for

$$
D^{a}\left(\varphi_{1}\right)=\frac{1}{2} \varepsilon^{a b c}\left[\varepsilon_{12} \partial_{b} \psi_{1} \partial_{c} \chi_{1}+\varepsilon_{21} \partial_{b} \chi_{1} \partial_{c} \psi_{1}\right] \neq 0, \quad a, b, c=1,2,3
$$

The theory of implicit functions proved that the solutions to Eqs. (18a) are given in the parametrical form as follows

$$
x=x_{\alpha}(s), y=y_{\alpha}(s), z=z_{\alpha}(s) \quad \alpha=1,2, \ldots, M .
$$

representing $M$ vortices of the first type. Analogously we have also $N$ vortices of the second type. They constitute a set of either contours or lines with two infinite extremities on the walls. We can check this statement by going along an arbitrary contour D rolling around a vortex $\mathrm{C}$ on the wall, one crosses the wall twice, the relative phase changes by $2 \pi$ (see Fig. 1). This is exactly the property of vortex.

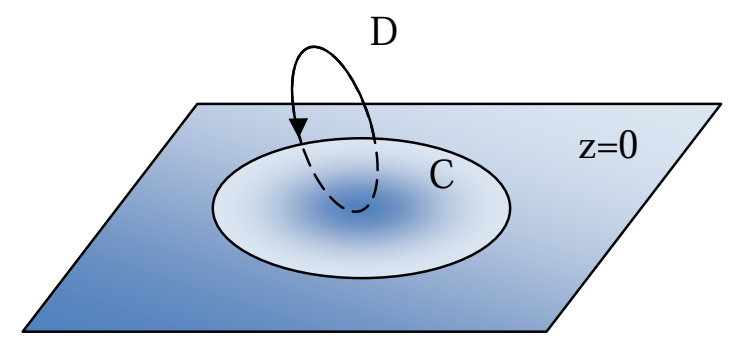

Fig. 1. Domain wall contains vortices 
Another property of domain wall can be extracted from Eqs. (20) and (21). Indeed, the currents of condensate densities are determined, respectively, by

$$
\vec{j}_{1}=u^{2} \nabla \alpha, \vec{j}_{2}=v^{2} \nabla \beta
$$

which combine together with (21) signify that the total currents of condensate densities vanish,

$$
\begin{gathered}
j_{1 z}+j_{2 z}=0, \\
j_{1 z}=4 k \frac{u^{2} v^{2}}{u^{2}+v^{2}} \frac{e_{z}}{\cosh k z}, \\
j_{2 z}=-4 k \frac{u^{2} v^{2}}{u^{2}+v^{2}} \frac{e_{z}}{\cosh k z}
\end{gathered}
$$

Eqs. (24) and (25) show that the domain wall is the plane $\mathrm{z}=0$ where two condensates flowing in opposite directions collide and then create two types of vortices with cores on the wall, see Fig. 2.

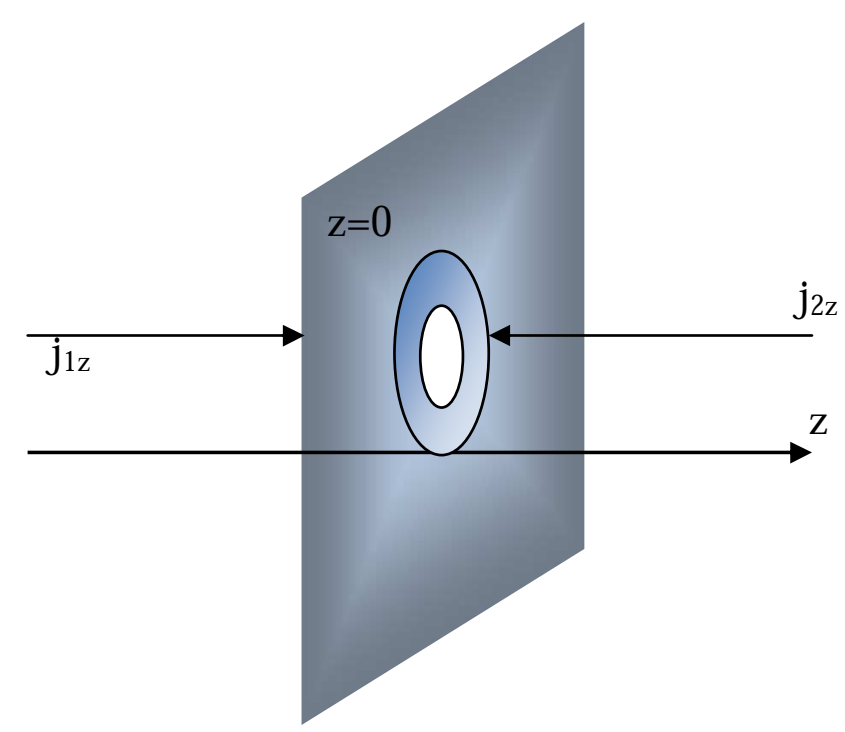

Fig. 2. Domain wall is the plane, where two condensates flowing in opposite directions collide and create vortices.

\section{5-Conclusion and Discussion}

In this paper we studied the physical properties of two interacting complex scalar fields at finite density and the main results we obtained are in order:

The Goldstone theorem does not realize in broken phase although the symmetry groups were spontaneously broken. Therefore the boson condensates do not become super fluids.

Under the influence of external magnetic field the condensates behave like superconductors. This fact is signaled by the appearance of the Meissner effect and magnetic vortices. 
Moreover, there exist two types of vortices, Abrikosov and non-Abrikosov vortices. They are trapped in domain wall which is the plane $\mathrm{z}=0$ where two condensates flowing in opposite directions collide and create two types of vortices with cores on the wall.

The next step is to consider not only the theory at finite density but also at finite temperature, then we are faced to the problem of phase transition of two-component Bose gases which is a very hot topic in recent years.

\section{REFERENCES}

[1] L. D. Landau and E. M. Lifshitz, Statistical Physics, Pergamon Press, NY 1969.

[2] P. G. de Gennes, Superconductivity of Metals and Alloys, W. A. Benjamin, INC. New York - Amsterdam, 1966.

[3] Quang Ho-Kim and Pham Xuan Yem, Elementary Particles and Their Interactions, Springer, 1998.

[4] C. Itzykson and J. B. Zuber, Quantum Field Theory, McGraw Hill International Editions, 1980.

[5] D. H. Lyth, hep-th/0311040.

[6] A. Linde, hep-th/0503195.

[7] A. M. Migdal, Sov. Phys. JETP 36, 1052 (1973).

[8] R. F. Sawyer, Phys. Rev. Lett. 29, 382 (1972).

[9] D. K. Campell, R. F. Dashen, and J. T. Manassah, Phys. Rev. D12 (1975) 979, ibid D12 (1975) 1010 .

[10] D. B. Kaplan and A. E. Nelson, Phys. Lett. B175 (1986) 57.

[11] M. G. Alford, K. Rajagopal, and F. Wilczek, Nucl. Phys. B537 (1999) 443 .

[12] J. B. Kogut and D. K. Sinclair, Phys. Rev. D64 (2001) 034508.

[13] J. B. Kogut and D. K. Sinclair, Phys. Rev. D70(2004) 094501.

[14] S. Gupta, hep-lat/0202006.

[15] A. Barducci et al, Phys. Rev. D71 (2005) 016011.

[16] L. He, M.Jin and P. Zhang, Phys. Rev. D71 (2005) 116001.

[17] L. He and P. Zhang, Phys. Lett. B615 (2005) 93.

[18] M. Frank et al, Phys. Lett. B562 (2003) 221.

[19] J. O Andersen and T. Brauner, Phys. Rev. D78 (2008) 014030.

[20] O. Scavenius et al, Phys. Rev. C64 (2001) 045202 .

[21] J. O. Andersen, Phys. Rev. D75 (2007) 065011.

[22] M. C. Birse et al, Phys. Lett. B516 (2001) 27.

[23] J. B. Kogut and D. Toublan, Phys. Rev. D64 (2001) 034007.

[24] M. Loewe and C. Villavicencio, Phys. Rev. D70 (2004) 074005.

[25] K. Splittorff et al., Phys. Rev. D64 (2001) 016003.

[26] Y. M. Cho, Phys. Rev. B72 (2005) 212516 and references herein.

[27] E. Babaev, Phys. Rev. Lett. 89 (2002) 067001.

[28] Y. S Duan et al., Nucl. Phys. B514 (1998) 705.

Received 01 January 2011. 